\title{
The Good, The Bad and The Ugly: Al in the higher education
}

\author{
Nino Popkhadze
}

\begin{abstract}
Higher education institutions face the Janus dilemma, on one hand, universities are asked to be more open, transparent, and easily accessible, so they can be better scrutinized by the public. On the other, they need to limit transparency and guard privacy.

The article explores how AI slowly but heavily penetrates the domain of higher education institutions; it provides various applications of $\mathrm{AI}$ in the domain of higher education. This paper argues that AI, big data, and learning analytics can become a powerful tool for advancing higher education institutions further, but at the same time, AI can have a detrimental effect without a vigilant eye. The paper does not aim to minimize the value and virtue of AI, rather problematize the implications and promote conscious decision-making. The article aims to stimulate discussion among the relevant stakeholders.
\end{abstract}

Keywords: AI, Big Data, Learning Analytics, Higher Education, Data-Driven Decisions

\section{INTRODUCTION: SETTING THE SCENE}

Higher education institutions face the Janus dilemma, on one hand, universities are asked to be more open, transparent, and easily accessible, so they can be better scrutinized by the public. On the other, they need to limit transparency and guard privacy. Therefore, there is a need for institutional intelligence, which will be the frontier of conscious decision-making, policy formation, and responsible data governance. This article is a discussion paper, intending to explore how Artificial Intelligence (AI) slowly but heavily disrupts the domain of the higher education institutions. The motivation for this paper stems from the possibilities and concerns this topic carries.
The paper argues that the weak signals of today may become wild cards in near future, it is foolish to disregard the power of AI, Big Data, and learning analytics. Thus, the article implies a warning message for higher education policymakers and practitioners to acknowledge the potential of AI and mitigate the risks. Besides, it is an invitation to take the pledge for conscious and responsible decision-making in the field of higher education. This paper does not aim to minimize the value and virtue of $\mathrm{AI}$, rather problematize the implications and promote conscious decision-making. The article aims to stimulate discussion among the relevant stakeholders.

Up to this point, AI and Big Data have penetrated institutional development, knowledge management, teaching, and learning within higher education institutions, they have promised to make respective processes more efficient and easier, and most importantly to make universities smarter or in fact, the smart universities (Lane 2014; Gagliardi 2018; Reidenberg \& Schaub 2018). Besides, it is believed that data analytics is an example of organizational innovation (Foss 2014). As Liebowitz $(2017,8)$ puts it "no matter where you turn, Big Data will have an impact. The education sector is no different". AI has emerged fast, but its deployment in higher education institutions is not ubiquitous yet, although it is a matter of time. In the AI Now Report, Campolo et al. (2017) recognized education as a "high stakes" domain, along with criminal justice, healthcare, and welfare, and recommended not to use "black box" AI and algorithmic systems.

The term of AI is connected with John McCarthy in 1950 when he organized a twomonth workshop at Dartmouth College in the USA and later used it in the proposal (Zawacki-Richter et al. 2019). The organization for Economic Co-operation and Development $(2019,7)$ has defined AI as "a machine-based 
system that can, for a given set of human-defined objectives, make predictions, recommendations, or decisions influencing real or virtual environments. AI systems are designed to operate with varying levels of autonomy". As Zawacki-Richter et al. $(2019,3)$ describe AI is not a single technology, rather "it is an umbrella term to describe a range of technologies and methods, such as machine learning, data mining, neural networks, or an algorithm". Current $\mathrm{AI}$ is known as narrow $\mathrm{AI}$, which does not exhibit the full range of intelligent and emotional characteristics similar to humans. Although the development of General AI is in the process, which will give AI self-awareness and consciousness (Zawacki-Richter et al. 2019; Southgate 2020). It is difficult to predict the future of AI, but scholars are sceptical about the development of General AI at this moment.

Big data represents massive data that cannot be handled with traditional hardware, therefore analysing big data is far more challenging. The main characteristics of big data are so-called $3 \mathrm{~V}$ : Velocity, Volume, and Variety (Kitchin 2014; Prinsloo \& Slade 2016). Kitchin $(2014,1)$ introduces other features: exhaustive in scope $(\mathrm{n}=\mathrm{all})$, fine-grained in resolution, flexibility, extensionality, relational, and scalability. Besides, Šman et al. (2020) add additional two more characteristics of $2 \mathrm{~V}$ : Veracity (data quality) and Value (value for business). To describe it easier with mundane words, big data is all about many bytes, the speed of gathering, and the variety of content. Speaking of bytes, big data is dealing with a larger number of storage such as terabytes, petabytes, zettabytes, and yottabytes. Šuman et al. $(2020,717)$ explain that big data can be structured, semi-structured or unstructured, but the essential feature is the size and diversity of the data, which leads to the challenges of storage, analysing, and retrieving. Although the more challenging part is how to use big data well and how to find the right value of the data. Deploying and using big data is known as machine learning.

Machine learning is a subfield of artificial intelligence, and it has become the main tool for leveraging data to identify patterns, predict and assist in decision-making. Southgate (2020) explains that machine learning is busy with developing algorithms that can learn from experiences. There are different types of machine learning: 1 . Supervised learning (data is labelled and guided by humans), 2. Unsupervised learning (algorithm creates own structure to detect patterns in unlabelled data), 3. Reinforced learning (algorithm interacts with a specific environment to make decisions, learns from trial and error). Yet another related concept is Deep learning, which is associated with artificial neural networks (ANN) to understand complex data, such as natural language processing (Southgate 2020,4).

The role of AI, Big Data, and analytics in the higher education context can be understood at micro, meso, and macro levels from academic and institutional perspectives (Daniel, 2017). At the micro level, higher education institutions deploy learning analytics to improve the teaching process and understand better patterns of students' behaviours. Learning analytics are illustrated as measuring, collecting, analysing, and reporting the learners' data to understand and optimize the learning environment (Siemens 2013, 1382; Klein et al. 2020). Khalil \& Ebner (2015) explain what constitutes big data in the higher education context, which is based on the learner's interaction with educational platforms. This type of interaction generates datasets that can be classified as following: Interaction data; Traces (number of logins, mouse clicks, and all types of digital activities with respect to educational platform); Personal data (name, address, email, and other types of information; Academic information (grades, learning journey, exams and so on). To get value from the data, special algorithms are applied and run, which is done through machine learning. On the meso and macro levels, institutions deploy robust models to enhance academic, institutional performance, effective decisionmaking, and accountability (Daniel 2017). The concrete examples of the application of AI in higher education will be discussed below.

The article has been organized in the following way: the overall structure of the paper takes the form of five sections. This section sets the scene and defines the main terms of AI, Big Data, Machine learning for common understanding. The second section - The Good describes the positive impact of $\mathrm{AI}$ in the higher education field and highlights current trends 
in this respect, such as personalized learning, improving academic success, and better insights for institutional development. The third section - The Bad accounts for the negative implications which stem from AI and learning analytics. This section problematizes the following aspects: profiling of the student, threatening freedom to learn, flawed algorithms, ethical and privacy issues. The penultimate section - The Ugly argues that $\mathrm{AI}$ and learning analytics may turn ugly and brings examples of surveillance, asymmetric power relationship, and data-driven decisionmaking, while posits that higher education is a moral practice with its values and virtues, thus, the human factor must not be minimized. The final section includes a summary and a brief discussion of the way forward. This article serves as the basis for further discussion, it tries to raise awareness about implications, challenges the status quo, and moots the importance of responsible decision-making within the higher education domain.

\section{THE GOOD}

The universities are a natural hotbed for big data as higher education is a human-rich system, built and driven by people. Consequently, by default, the universities used to keep the record and store the data. Prinsloo \& Slade (2016) hold a similar view and observe, that historically higher education has always had access to student data and used it for institutional planning, before learning management systems they could rely on aggregated student data. Thus, the novelty is the digitalization of the data and its computational capacity, which allows the higher education institutions to apply machine learning and learning analytics, and study not only aggregated student data but rather observe individual student's performance as well.

Zawacki-Richter et al. (2019) conducted a systemic review and researched the application of $\mathrm{AI}$ in higher education quite extensively. Within the study, the student life-cycle framework was applied to portray AI-based services. The authors distinguish various AI applications, such as profiling and prediction, admission decisions and course scheduling, drop-out and retention, student models and academic achievement, intelligent tutoring systems, automated grading, feedback, recommending/providing personalized content, supporting teachers in learning and teaching design, assessment, and evaluation, using academic data to monitor and guide students, adaptive systems, and personalization. Zawacki-Richter et al. (2019) illustrate diverse pedagogical opportunities to enhance student learning in an adaptive and personalized manner. Additionally, it is argued that AI may solve the problem to provide mass education, and through automation burden of professors will be reduced.

Learning analytics and educational data mining can draw the bigger picture on an institutional level and provide trends related to academic programs and staff (Reidenberg \& Schaub 2018). Learning analytics tools can automate some teaching elements, such as advising, assessment, feedback to personalize learning, encourage and alert students regarding their learning behaviours (Klein et al. 2020). Furthermore, as Siemens (2013) describes, learning analytics heavily feeds from two sources: student information systems (SIS) and learning management systems (LMS). The former helps to generate a student's profile, and the latter represents one of the technological tools which analyses the data and sends warning signals regarding the student's performance, course retention, and so on.

Siemens $(2013,1388)$ differentiates between learning analytics techniques (modelling, relationship mining, knowledge domain modelling) and applications (trend analysis and prediction, personalization/adaptive learning, structural analysis). For the scope of this paper, the modelling technique is interesting as it enables user profile development, behaviour, and learner modelling. Besides, equally interesting is an early warning and risk identification, measuring the impact of interventions and changes in learner behaviour which fall under the application of trend analysis and prediction.

Howell et al. (2017) underline that many higher education institutions apply learning analytics to predict student retention, to understand and improve student behaviour, and to provide personalized feedback and support. The research undertook by Howell et al. (2017) voiced academics, their attitudes, and pedagogical concerns regarding learning 
analytics in the Australian context. The value seen in learning analytics by academics is focusing on at-risk students to understand the factors and enhance learning.

Roberts et al. (2016, 91) distinguish the benefits for students and the higher education institutions, for students learning analytics provide insights into learning habits, detecting at-risk students and intervening at an early stage, and providing a personalized learning experience. The value for universities lies in competitive advantage through data-driven decision-making to increase organizational productivity and effectiveness. Khalil \& Ebner (2015) summarize the capacity of learning analytics:

- Prediction: From the available datasets the algorithm analyses student's academic performance and predicts the future.

- Intervention: Again, through feeding on the data, an algorithm is capable to prevent dropouts, assist at-risk students to support their academic success.

- Recommendation: Similar to business intelligence, learning analytics are capable to recommend educational products (books, programs, lectures) based on preferences and interests.

- Personalization: Learning analytics are mostly promoted and applied because they enable personalized learning, which hitherto was problematic. The learner can shape a distinctive learning environment, consequently, suggestions will be customized to her/his ability and preference.

- Reflection and Iteration: This helps learners to track and improve their progress and performance.

- Benchmarking: learning analytics are programmed to find the best practices as a reference point, while comparison gives the possibility to detect weak points of educational performance and generates suggestions to enhance learning and learner's success.

Gagliardi (2018) describes the modern period as the analytics revolution, big data provides an array of possibilities for higher education to satisfy the demand. It is portrayed as an institutional and campus-wide affair, as it is related to student success, cost-saving endeavours, and gathering evidence. The analytics revolution enabled the transformational shift from descriptive data reporting towards more prescriptive and predictive data analytics.

Goff \& Shaffer (2014) explore the big data impact on university admission and recruitment process, and the way it improves overall efficiency. They argue that data-informed and informationdriven decisions became an integral part of strategic enrolment management. Richer data analytics can forecast students' interest, the likelihood of persisting to graduation, financial need levels, successfully passing courses, and so on. It is underlined that higher education institutions "are sitting on literally mountains of data" because of learning platforms, campus behaviour, and portal activity, and yet not all institutions translate the data into actionable information for decision-making (Goff \& Shaffer 2014, 96).

As Gagliardi (2018) puts it, robust data analytics has been considered a major ingredient in strategic innovation and it should be regarded as an institutional asset. Webber \& Zheng (2020) explain the concept of Data-Informed Decision Making as the process which looks at the data as one of the tools to get evidence and provide respective strategy, in this case, data is guiding and auxiliary. Data-informed decision-making enables the university to meet external demand and expectations, create new academic programs, support student's success, and strengthen links with industry (Webber \& Zheng 2020).

Optimal use of student-generated data allows higher education institutions to make better and informed choices and respond to changes faster (Slade \& Prinsloo 2013). Mathies (2018b) posits few examples as predicting the enrolment numbers and identifying the focused admission recruitment based on the previous data. In fact, whether someone is Luddite or a tech enthusiast, it is without a shadow of a doubt that big data and learning analytics are promising phenomena to optimize processes in various fields, and higher education is one of them.

It is a promising trend that privacy and ethical use of data started to attract attention recently, which slowly awoke policymakers in that regard, for instance, European Union (2016) has adopted the General Data Protection 
Regulation (GDPR) in 2016 which is a positive move forward. The ongoing project "Fostering Fair Data Practices in Europe - FAIRsFAIR started in 2019 to supply practical solutions to use fair data principles throughout the research cycle in Europe. Roberts et al. $(2016,97)$ draw attention to the importance of data governance, which entails four elements: accountability, transparency, predictability, and participation to ensure healthy mechanisms for data ownership and endorse trust from stakeholders. It is deemed vital for higher education institutions to be confident with data quality, ethics, privacy, and security. Although regulations, guidelines, and frameworks do not work on their own, they need to be applied and translated into institutional practices, which calls attention to professionals who know the technology (information technology, analytics) and have special expertise to optimize the results of the data for institutional development.

One of the examples is institutional research (IR) which is fairly considered as organizational intelligence (Terenzini 1993) and the "center of gravity of all of the university's analytical and decision support activities" (Volkwein et al. 2012 38). Baldasare (2018) describes IR offices' experience as decision support units through predictive modelling, forecasting, statistical analysis, ad hoc, and standard reports. In the light of deep learning, institutional researchers have the capacity for institutional-wide learning and diagnosis. As Slade \& Prinsloo (2013) argue, higher education cannot afford not to use learning analytics, HEIs should capitalize on data analytics, but responsibly and consciously.

\section{THE BAD}

While supporting student's learning journey or improving institutional processes do sound noble and error-free, educational data mining is not a linear process, and some factors need to be considered. The first question remains to what extent learning analytics supports and enhances student's learning, and to what extent it threatens academic autonomy.

\section{Freedom to learn (Lernfreiheit)}

Learning analytics can be looked at from two angles, the one which enables learning and the other, which intrusively shadows the learner. Going back to Wilhelm von Humboldt, who made a fundamental shift in the understanding of universities and introduced the notion of academic freedom (Kerr 2001), this paper argues that especially freedom to learn (Lernfreiheit) is endangered. Beattie et al. $(2014,423)$ question the academic freedom and autonomy of learners, when learning relationships are dictated behind the black box created often by the corporate outsiders.

Learning analytics contributes to patronizing student's learning experience which leads to moulding, stereotyping, and labelling the students. Roberts et al. $(2016,92)$ discuss that the aspect of so-called failing students is important, as the array of negative comments can easily influence the student's well-being, self-efficacy, and self-perception, it may have the effect of "self-fulfilling prophecy" in the end. Also, the interesting part of this discussion is the promise of more personalized learning, which is carried out, in fact, at the expense of homogeneity and leads to the linear understanding of student's success that only high achieving students equal to the most successful ones. This could be seen from an institutional perspective, as retention rate, completion and cohort graduation rate are seen also as institutional success (Guibault 2016). By contrast, Slade \& Prinsloo (2013, 1520 ) assert that student success is a complex and multidimensional phenomenon, which represents interdependent interaction among students, institutions, and societal factors.

The research conducted by Howell et al. (2017) brings to the fore the patronizing element of learning analytics and poses the challenge of becoming a "Helicopter University". They describe the situation when the algorithm pushes students in a direction that may not be in their best interest and students should have the freedom to change their minds and craft their learning journeys independently, which may even lead to changing professions or the university. The "one system fits all" approach is also criticized to the point that moulding sets unrealistic expectations and definitions 
of academic success. Therefore, it is expected that learning analytics, inclusiveness, and individualism should go together, but in practice, it promotes one standard for all. Concerning the notion of freedom to learn, the learners' responsibility for their learning process constitutes another noteworthy point, whether the active learning principle is impeded or not when decisions are made by AI (Howell et al. 2017).

Reidenberg \& Schaub (2018) depict the danger of false positive when the algorithm indicates a lack of engagement from the learner, although the problem is the flawed code. Thus, instead of improving or optimizing the student's learning experience, it most likely will be deteriorated. Prinsloo \& Slade $(2016,116)$ also suggest that there is the possibility that "algorithms may increase the vulnerability of individuals through stigmatization and special tracks". Furthermore, Webber \& Zheng (2020) argue that even though learning analytics are maturing, there is little evidence that it improves student outcomes.

\section{Ethics \& Privacy}

Ethical and privacy issues are emerging research phenomena and AI implications in higher education (Siemens 2013, Beattie et al. 2014; Khalil \& Ebner 2015; Slade \& Prinsloo 2013; Prinsloo \& Slade 2016; Mathies 2018a; Mathies 2018b; Reidenberg \& Schaub 2018; Klein et al. 2020) and deserve special attention. Reidenberg and Schaub $(2018,1)$ eloquently and reasonably compared education, big data, and student privacy to a combustible mixture. As it was mentioned earlier, higher education institutions are a cradle for big data, which brings opportunities and poses several risks regarding ownership, privacy, and ethical use of big data.

The challenge with privacy occurs when higher education institution discloses and exchanges student or employee personal data to the third party, without prior consent. Mathies (2018b, 7-8) criticizes the misuse of the data in higher education and describes the case of the University of Oregon, in which the student filed a lawsuit against the university. Later, student's mental health records were accessible to the university's lawyer to defend the university. The case caused discussions and changes regarding the protection of students' health records in the state of Oregon.

\section{Data Governance}

$\mathrm{AI}$ and digitalization made it possible to become digitally loud, as every move or click counts and is stored, thus individuals are more exposed to malfeasance and data breaches (Beattie et al. 2014). As Siemens (2013) posits privacy and data ownership are not unique concerns to analytics, as any type of digital interaction leaves the footprint. Thus, the questions remain who owns data, who has access and how long the university should keep the data, in search of answers, responsible data governance becomes crucial. The collection of student information carries an inherent risk for privacy, and violation of privacy may cause distrust in the education institutions (Reidenberg \& Schaub 2018).

Reidenberg \& Schaub (2018) address the integrity of the data when students exclude their data from usage and its ramification for learning analytics to become potentially incomplete and skewed. Furthermore, data analytics enables to treat data as a commodity for corporate purposes as it can have an economic value (Howell et al. 2017). Therefore, there is the risk of function creep, when data is collected for one purpose, but it is used for the other as well (Southgate, 2020). Beattie et al. $(2014,422)$ argue that data should not be sold off without the owner's permission and warn of the dual danger of proposing learner data rights, when naïve learners may agree through one click without caution, and security-savvy learners may abstain from data analytics. Roberts et al. (2016) reinforce the same idea with researchbased evidence and argue that when students consent to learning analytics upon enrolment may not be aware of what it really means.

The algorithms drive institutional-wide decisions, many higher education institutions use algorithms for admission purposes. Mathies (2018a) suggests that when the admission formula is getting updated, it most likely leads to coding failure. Thus, flawed code may be unnoticed unless a large group of people is affected. Yet another example when flawed code becomes problematic is lending the loans for 
students. Mathies (2018a, 89) argues that during a highly competitive admission process, risk assessment algorithm most likely will favour the candidates with advanced placements, hence students who come from low-resourced schools have disadvantage positions and chances are high they will get rejected in comparison with their peers.

Mathies (2018a) posits that the concept of ethics in data governance has a degree of relativity, as what is considered ethical in one situation may not be in another, therefore an ethical framework is necessary as a guiding principle. Similarly, Prinsloo \& Slade (2016) moot the concept of "Ethics of Care", which is rather value-driven and implies relational understanding in harvesting, analysis, and use of data.

\section{THE UGLY}

Under this section, it is believed that data analytics and a data-driven approach may get ugly. Several scholars have described the context regarding AI and higher education as the following epithets "dark side" (Siemens 2013, 1395), "creepy analytics" (Beattie et al. 2014), "operate out of darkness" (Mathies 2018a, 85).

\section{Surveillance \& Trust}

One distinctive power of learning analytics is surveillance, as Beattie et al. $(2014,421)$ put it "we know what students are thinking before they even think it". Reidenberg \& Schaub (2018) posit that fear of surveillance and tracking of student behaviour may result in chilling effects. One of the examples is special cameras harnessed at the campus to collect geolocation and biometric data, such as facial and voice recognition, fingerprints, body temperature, heart rate, stress level, gaze attention, and other types of behavioural information (Reidenberg \& Schaub 2018; Zawacki-Richter et al. 2019; Southgate 2020). Southgate (2020) raises important issues regarding biometric data and the human right to bodily integrity.

Knox (2010) explores the issue of surveillance quite extensively from the perspective of the Online Learning Environment (OLE). $\mathrm{He}$ distinguishes between surveillance and monitoring, all surveillance involves monitoring, but not vice versa. For illustration, Knox (2010, 5-7) brings Michel Foucault's theory of the Panopticon from automation and visibility angles. The discourse is applied in the education context as following when students $\log$ in, they are visible for the system, although the tracking process is invisible for the learner, which leads to relationship structures, trust, and power dynamics between learners and authority (instructor and university management). Knox (2010) asserts that the corrosion of trust is a salient matter for higher education, and it should be a primary concern for the lecturers and students while using an online learning environment.

Similarly, Slade \& Prinsloo (2013) approach learning analytics as power relationships between learners, higher education institutions, and other stakeholders, and relate to Foucault's perspective of the Panopticon, as an authority has the power to control all activity. This simply means that the university as an authority (university leadership, data custodian, broker, instructor) has access to students' information, while the student doesn't have the same possibility. There is a tendency that students alter their online behaviour when they are aware of institutional surveillance (Slade \& Prinsloo 2013). Therefore, Prinsloo \& Slade $(2016,120)$ assert also that higher education carries a "fiduciary duty" to its students, especially when there is an asymmetric relationship, thus all decisions must embody justice and care. Similarly, Beattie et al. (2014) posit that any technological changes can rupture trust among learners, teachers, and institutions. It is important to remember that students are active co-creators of their learning journey and should be informed regarding privacy issues with due diligence. The students should have the right to negotiate to what extent they want to be surveyed and monitored, as Slade \& Prinsloo (2013) suggest informed consent in higher education is almost as important as a sine qua non.

\section{Data-Driven Decision-Making}

In line with the ongoing discussion of knowledgedriven and data-driven science (Kitchin 2014), another peril for higher education is an approach 
of Data-Driven Decision-Making. Webber \& Zheng (2020) explain this latter as a decision process driven by algorithms while the human factor remains minimized. The wave of data mining is coming from the business community (Baldasare 2018), thus similar to the business community there is pressure from data analytics revolution for the higher education field, which sometimes leads to the situation that processes are data rich but information poor (Webber \& Zheng 2020). Some of the tasks can be easily automated, for instance, to remind students to pay fees before the deadline or evaluate student's eligibility for degree completion. Although the question remains to what extent the decision process should be data-driven on the institutional levels. If the higher education institution is guided solely by data, there is a possibility it loses the sense of the environment, its people, and mission. And if universities and their priorities are driven by data and not by humans, then how does it shape the future of the universities?!

The learning analytics analyses the symbols and shows the trends based on the source which can be quantifiable. At the same time, it must be remembered that as Clark $(1983,240)$ puts it higher education institutions have inherent system values: justice, competence, liberty, and loyalty. If data becomes the sole manipulator of the decision process, there is a serious threat to neglect these values and other social, cultural, or political trends that cannot be analysed through raw data. Thus, higher education institutions need strategic planning which goes beyond the data, data will not remind universities of its values, virtues, and guiding principles. Going back to one of the ultimate missions of the university, to serve society and to tackle societal problems, perhaps it is time to bring to the fore the original discussion of "Wicked Problems". Rittel \& Webber (1973) contend that there are two types of problems, tamed and wicked ones. The former is explained through the example of scientists, mathematicians, and engineers, as it is clear when they solve a problem, for instance, an equation or while playing chess. The latter does not have clarity, there are no true and false answers, and each solution generates waves of consequences, which cannot be undone. For this type of problem, there is no opportunity to learn from trial-and-error, every attempt to solve the problem counts and is irreversible, thus it is considered inherently wicked.

Higher education institutions operate to tackle both types of problems, and especially to solve complex and wicked problems, therefore human factors should not be compromised in the decision process. Slade \& Prinsloo (2013, 1519) points to the fact that learning analytics should not only focus on effectiveness but rather "should function as a moral practice". The same idea is reinforced by Webber \& Zheng (2020) while they moot that data-informed decisionmaking is rather balanced way as it reflects the dynamic environment, embraces human judgment, and acknowledges that data is an invaluable source for decision insights, at the same time, data is not perfect and error-free. Ex-ante damage control is necessary, as higher education institutions are a high-risk domain and they should not be driven by trial-anderror bases. Slade \& Prinsloo $(2013,1514)$ posit that despite the inherent promises that learning analytics enables institutions to have it all, in the end, all institutions must decide what is the main purpose, whether to maximize the number of students who graduate, to support those in need, or to maximize the profit, otherwise it may lead to broken promises.

\section{CONCLUSION}

In the spirit of the current time, we live with the fast-evolving landscape of AI, at this moment known as Narrow AI, whereas working on General AI is underway, thus the power of AI is still not fully gauged and its imprint on higher education will only be intensified. There are great things to achieve through $\mathrm{AI}$ and there are great things to risk. The data-driven approach awakes the sore discussion about losing the soul of universities, which deserves to be addressed further.

As it was mentioned earlier, big data is equal to the big number of bytes, and subsequently, this leads to unseen, yet significant environmental impact. Therefore, clear guidelines are necessary to understand what type of data should be stored and secured, how long, where, and when to get rid of it. There should be a common language to classify data according to high-low risk, besides 
storage of the data, and environmental aspects of it will be part of future research and inquiry.

It falls outside the scope of this paper to discuss the solutions to tackle the abovementioned implications, but many scholars are already discussing the way forward to keep a vigilant eye. $\mathrm{AI}$ in the higher education context continues to be an area worthy of research, there is and will be continued discussion, as it is a topic that must allow reflection and critique. It is impossible to cover all aspects and implications of AI in higher education within one paper, but the relentless effort must be sustained to explore the ethical and responsible use of AI in higher education further and provoke dialogue among various stakeholders. In the current period, we live under the intersection of AI romanticism and Luddites, tech enthusiast and tech sceptics,

\section{REFERENCES:}

Baldasare, Angela Y. (2018). Pursuit of Analytics and the Challenge of Organizational Change Management for Institutional Research. In Swing, Randy L., Parnell, Amelia R., Gagliardi, Jonathan S. \& Carpenter-Hubin, Julia (Eds.), The analytics revolution in higher education: big data, organizational learning, and student success ( $1^{\text {st }}$ ed., 71-88). Stylus Publishing LLC.

Beattie, Scott, Woodley, Carolyn \& Souter, Kay (2014). Creepy analytics and learner data rights. Proceedings of ASCILITE 2014 - Annual Conference of the Australian Society for Computers in Tertiary Education, 421-425.

Campolo, Alex, Sanfilippo, Madelyn, Whittaker, Meredith \& Crawford, Kate (2017). AI Now 2017 Report. New York: AI Now Institute. Retrieved from https://ainowinstitute.org/AI Now_2017_Report.pdf

Clark, Burton (1983). The higher education system : academic organization in cross-national perspective. University of California Press. https://doi.org/10.1525/9780520340725

Daniel, B. K. (2016). Big Data in Higher Education: The Big Picture. In Daniel Ben K. (Ed.), Big Data and Learning Analytics in Higher Education (pp. 19-28). Springer International Publishing. https://doi.org/10.1007/978-3-319-06520-5_3

European Union (2016). General Data Protection Regulation (GDPR) - Official Legal Text. Retrieved from https://gdpr-info.eu/

FAIRsFAIR (2019). The Project | Fostering FAIR Data Practices in Europe. Retrieved from https:// www.fairsfair.eu/the-project thus, more than ever is crucial to ask critical questions and adopt responsible stand. It is paramount to question the added value of every technological intervention in the pedagogical and institutional processes, as technologically many things can be possible nowadays, but that is not the main point.

This article aimed to explore challenges and invigorate further discussion. It is foolish to disregard the benefits AI brings and will bring to higher education institutions, but it must be remembered that higher education is the domain to do the right things and not just things right. It should not be forgotten that AI was coined in the realm of higher education, therefore, it is only fair to request to apply its legacy in an ethical, responsible, and meaningful fashion.

Foss, Lisa H. (2014). Integrating Data Analytics in Higher Education Organizations: Improving Organizational and Student Success.In Lane Jason E. (Ed.), Building a Smarter University: Big Data, Innovation, and Analytics (pp. 187209). State University of New York Press.

Gagliardi, Jonathan S. (2018). The Analutics Revolution in Higher Education. In Swing, Randy L., Parnell, Amelia R., Gagliardi, Jonathan S. \& Carpenter-Hubin, Julia (Eds.), The analytics revolution in higher education : big data, organizational learning, and student success ( $1^{\text {st }}$ ed., pp. 1-14). Stylus Publishing, LLC.

Goff, Jay W. \& Shaffer, Christopher M. (2014). Big Data's Impact on College Admission Practices and Recruitment Strategies. In Lane Jason E. (Ed.), Building a Smarter University: Big Data, Innovation, and Analytics (pp. 93-120). State University of New York Press.

Guilbault, Melodi (2016). Students as customers in higher education: reframing the debate. Journal of Marketing for Higher Education, 26(2), 132142. https://doi.org/10.1080/08841241.2016.12 45234

Howell, Joel A., Roberts, Lynne D., Seaman, Kristen \& Gibson, David C. (2018). Are We on Our Way to Becoming a "Helicopter University"? Academics' Views on Learning Analytics. Technology, Knowledge and Learning, 23(1), $1-20$. https://doi.org/10.1007/s10758-017-9329-9

Khalil, Mohammad \& Ebner, Martin (2015). Learning Analytics: Principles and Constraints. 
In Proceedings of World Conference on Educational Multimedia, Hypermedia and Telecommunications 2015. pp. 1326-1336. Chesapeake, VA: AACE.

Kitchin, Rob (2014). Big Data, new epistemologies and paradigm shifts. Big Data \& Society, 1(1). https://doi.org/10.1177/2053951714528481

Klein, Carrie, Lester, Jaime, Rangwala, Huzefa \& Johri, Aditya (2020). Learning Analytics for Learning Assessment: Complexities in Efficacy, Implementation, and Broad Use. In Karen L. \& Zheng, Henry Y (Eds.), Big Data on campus: Data-informed decision making in higher education. Johns Hopkins University Press.

Knox, Dan (2010). A good horse runs at the shadow of the whip: Surveillance and organizational trust in online learning environments. Canadian Journal of Media Studies. Retrieved from http://cjms.fims.uwo.ca/issues/07-01/ dKnoxAGoodHorseFinal.pdf

Lane, Jason E. (2014). Building a Smarter University: Big Data, Innovation, and Analytics. State University of New York Press.

Liebowitz, Jay (2016). Thoughts on Recent Trends and Future Research Perspectives in Big Data and Analytics in Higher Education. In Daniel Ben K. (Ed.), Big Data and Learning Analytics in Higher Education (pp. 7-17). Springer International Publishing. https://doi. org/10.1007/978-3-319-06520-5_2

Mathies, Charles (2018a). The Ethical Use of Data. New Directions for Institutional Research, 2018(178), 85-97.

https://doi.org/10.1002/ir.20269

Mathies, Charles (2018b). Uses and misuses of digitalis. In Webber Karen l. (Ed.), Building Capacity in Institutional Research and Decision Support in Higher Education (pp. 95-111). Knowledge Studies in Higher Education 4. https://doi.org/10.1007/978-3-319-71162-1_7

OECD. (2019). Recommendation of the Council on Artificial Intelligence . https://legalinstruments. oecd.org/en/instruments/OECD-LEGAL-0449

Prinsloo, Paul \& Slade, Sharon (2016). Big Data, Higher Education and Learning Analytics: Beyond Justice, Towards an Ethics of Care. In Daniel Ben K. (Ed.), Big Data and Learning Analytics in Higher Education (pp. 109-124). Springer International Publishing. https://doi.org/10.1007/978-3-319-06520-5_8

Reidenberg, Joel R. \& Schaub, Florian (2018). Achieving big data privacy in education. Theory and Research in Education, 16(3), 263-279. https://doi.org/10.1177/1477878518805308

Rittel, Horst W. J. \& Webber. Melvin M. (1973). Dilemmas in a General Theory of Planning.
Policy Sciences, 4(2), 155-169.

https://doi.org/10.1007/BF01405730

Roberts, Lynne D., Chang, Vanessa \& Gibson, David C. (2016). Ethical Considerations in Adopting a University- and System-Wide Approach to Data and Learning Analytics. In Daniel Ben K. (Ed.), Big Data and Learning Analytics in Higher Education (pp. 89-108). Springer International Publishing. https://doi.org/10.1007/978-3-319-06520-5_7

Siemens, George (2013). Learning Analytics: The Emergence of a Discipline. The American Behavioral Scientist (Beverly Hills), 57(10), 1380-1400. https://doi.org/10.1177/0002764213498851

Slade, Sharon \& Prinsloo, Paul (2013). Learning Analytics: Ethical Issues and Dilemmas. The American Behavioral Scientist (Beverly Hills), 57(10), 1510-1529. https://doi.org/10.1177/0002764213479366

Southgate, Erica (2020). Artificial Intelligence, ethics, equity and higher education: A beginning-of-the-discussion' paper. National Centre for Student Equity in Higher Education, Curtin University, and the University of Newcastle

Šuman, Sabrina, Poščić, Patrizia \& Marković, Maja G. (2020). Big Data Management Challenges. International Journal of Advanced Trends in Computer Science and Engineering, 9(1), 717723.

https://doi.org/10.30534/ijatcse/2020/102912020

Terenzini, Patrick T. (1993). On the nature of institutional research and the knowledge and skills it requires. Research in Higher Education, 34(1), 1-10. https://doi.org/10.1007/BF00991859

Volkwein, Fredericks J., Liu, Ying J. \& Woodell, James (2012). The structure and functions of institutional research offices. In Howard Richard D., McLaughlin Gerald W. \& Knight WILLIAM E. (Eds), The handbook of institutional research (pp. 22-39). Hoboken, N.J. John Wiley \& Sons.

Webber, Karen L. \& Zheng, Henry Y. (2020). Chapter 1-1. In Webber, Karen L. \& Zheng, (Eds.), Data analytics in higher education (pp. 1-33). Johns Hopkins University Press.

Zawacki-Richter, Olaf, Marín, Victoria I., Bond, Melissa \& Gouverneur, Franziska (2019). Systematic review of research on artificial intelligence applications in higher education - where are the educators? International Journal of Educational Technology in Higher Education, 16(1), 1-27. https://doi.org/10.1186/s41239-019-0171-0 